\title{
Massive Radio Channel Sounder Architecture for 5G Mobility Scenarios: MaMIMOSA
}

\author{
P. Laly*, D.P. Gaillot* ${ }^{*}$, G. Delbarre*, M. Van den Bossche ${ }^{\ddagger}$, G. Vermeeren ${ }^{\ddagger}$, F. Challita*†, E. Tanghe ${ }^{\ddagger}$, \\ E.P. Simon ${ }^{* \dagger}$, W. Joseph ${ }^{\ddagger}$, L. Martens ${ }^{\ddagger}$, M. Liénard* \\ *IEMN (UMR 8520), University of Lille, Villeneuve d'Ascq, France. martine.liénard@univ-lille.fr \\ †IRCICA (USR 3380), University of Lille, Villeneuve d’Ascq, France. davy.gaillot@univ-lille.fr \\ $\ddagger$ INTEC - WAVES, Ghent University, Ghent, Belgium. wout.joseph@ugent.be
}

\begin{abstract}
This paper presents a real-time $64 \times 16$ massive MIMO channel sounder based on space-frequency division multiplexing and antenna subarray switching, giving a large possibility of antenna allocation and frequency tone between 2 and 12 GHz. This channel sounder called Massive MIMOSA or MaMIMOSA belongs to the new generation of software radio design based systems. The architecture of the proposed approach was designed with the highest flexibility thus opening a wide range of applications depending upon the investigated scenarios. Currently, the system is being setup using a massive $10 \times 10$ antenna array operating around $6 \mathrm{GHz}$ with $80 \mathrm{MHz}$ bandwidth for 5G V2X applications. The channel sounder can be powered with vehicle batteries to perform day long measurements when electrical outlets are not available. The output file gives the measured Massive MIMO matrix in a friendly compact binary format. It will be operational in early 2020 .
\end{abstract}

Index Terms-Massive MIMO channel sounding, V2X, propagation, measurements.

\section{INTRODUCTION}

Mobile connectivity has become not only essential but a necessity for many network users. Technological advances and computer abilities are needed to provide faster, smarter and safer wireless networks [1]. The domain of application is wide and not limited to mobile devices and cellular networks [2] but now also includes connected machines in industrial setups, vehicular communications (vehicle-to-everything or V2X) [3] and smart cities [4], [5]. The upcoming 5G NR (New Radio) being currently defined by 3GPP [6], [7] is projected to supply unprecedented data rates with improved networks efficiency. The main envisioned use cases to be supported span three different dimensions: enhanced mobile broadband (eMBB), massive machine type communications (mMTC) and ultrareliable low latency communications (URLLC). Promising technologies capable of fulfilling the gap from previous generations are sought such as Massive MIMO [8] and the use of larger bandwidths at millimeter wave $(\mathrm{mmW})$ frequencies. It is now acknowledged that massive MIMO is a form of MU-MIMO, an asymptotic extension where the number of antennas $\mathrm{M}$ is very large and many user equipments (UE) are simultaneously served. Generally speaking, the receivers (UEs, machines, industrial robots, etc.) in $5 \mathrm{G}$ use-cases are equipped with one antenna [9]-[11]. The basic information, theoretical aspects and limits were presented in early works such as [12], [13].
Hence, propagation models generally used in network planning tools must be revisited and tuned for the new $5 \mathrm{G}$ massive MIMO technologies and frequency ranges. In addition, the main benefits envisioned by massive MIMO such as the reduction in latency on the air interface as well as the simplification of the multiple-layer access due to channel hardening or even robustness to undesired or intentional jamming [14] must be evaluated. Moreover, from the Tx array side, spatial and temporal stationarity assumptions of the channel are not often verified in realistic dynamic environments. For instance, the current radio models rely on the hypothesis of the wide-sense stationarity (WSS) uncorrelated scattering (US) assumption [?]. The WSSUS assumptions imply that second order channel statistics are independent of time and frequency, and hence, allow for a simplified statistical description of channels; this has formed the basis of many designs of wireless transceivers. However, the WSSUS assumptions are not always fulfilled in practice, particularly in vehicular scenarios [15] and dedicated radio channels must be clearly revisited with real-time massive MIMO systems. Hence, a massive MIMO system with a fully physical M-antenna array connected to M RF chains or less is a requisite to grasp the time-varying spatial characteristics of the radio channel. However, due to the complex architecture, this approach can be rather costexpensive especially if each radiating element is connected to an independent RF chain [16] and can result in RF impairments or synchronization issues between the massive array and the UEs.

To this end, a massive MIMO radio channel sounder operating between 2 and $12 \mathrm{GHz}$ with $80 \mathrm{MHz}$ bandwidth has been jointly developed by the University of Lille (France) and Ghent (Belgium) for 5G mobility scenarios such as V2X communications. The architecture relies on the physical and reconfigurable software radio channel sounder MIMOSA [17] which is capable of performing real-time measurements at $1.35 \mathrm{GHz}$ like reported in [15] to analyze the stationarity of V2I radio channel in a Suburban Environment. MaMIMOSA has been designed to fulfill all the constraints identified in time-varying massive MIMO channels such as high Doppler resolution with a large Doppler span, spatial antenna selection, compactness, energy consumption, etc. The sounding parameters of the developed massive system can be freely selected depending on the studied environment resulting in the 
measured massive MIMO radio channel without the need for additional tedious post-processing. As of now, MaMIMOSA is being setup to perform $64 \times 16$ massive radio channel measurements at $5.89 \mathrm{GHz}$ for $\mathrm{V} 2 \mathrm{X}$ communications. The 1.3 GHz frequency of MIMOSA is used as an intermediate frequency (IF) to transpose the baseband signal to RF at 5.89 $\mathrm{GHz}$ with $80 \mathrm{MHz}$ bandwidth. This paper describes the main features and architecture of MaMIMOSA which is currently under progress and will be fully operational in early 2020 . Preliminary results will be presented during the conference.

\section{Channel Sounder Architecture}

\section{A. Frame structure}

The basic 64 x 16 Massive MIMO transmitting frame structure consists in one $51.2 \mu$ s preamble subsequently followed by 8 OFDM symbols of $121.92 \mu$ s including the cyclic prefix as illustrated in Fig. 1. Hence, the total OFDM sequence to measure a full $64 \times 16$ massive MIMO matrix is $\sim 1 \mathrm{~ms}$. The preamble provides the time synchronization of the frame such that the OFDM symbols can be decoded. Each OFDM symbol corresponds to an 8-antenna subarray out of 64 and the OFDM sequence (i.e. subarray sequence) can be selected by the user. The time duration between consecutive frames can be manually set from $1 \mathrm{~ms}$ to any larger values depending on the desired maximum Doppler shift. As initially developed for MIMOSA [17], the total number of transmitted OFDM subcarriers per symbol $N_{t}$ is 8192 which can be uniformly distributed on each antenna element using interleaved OFDM. For instance, the frequency space $\delta F$ between the subcarriers of a single antenna is $97.66 \mathrm{kHz}$, meanwhile the frequency space of between subcarriers is $12.21 \mathrm{kHz}$. The automatic gain control (ACG) is performed with the cyclic prefix for each symbol and is used to correct the measured MIMO matrix.

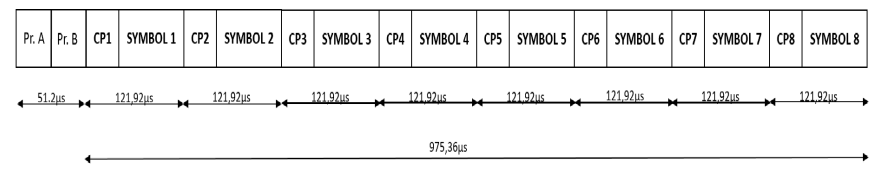

Fig. 1. MaMIMOSA frame structure.

\section{B. Transmitter and Receiver Unit}

1) Tx Unit: The MaMIMOSA transmitter (Tx) chain is presented in Fig. 2. It is composed of 4 parts: 1) "time and position" records information such as the GPS position and $3 \mathrm{D}$ position of the antenna array (yaw, pitch, roll). The reference rubidium clock is also found to synchronize all Tx signals. 2) The signal manager is organized thanks to a computer embedded into a chassis which also includes the FPGA-based digital processing cards to generate and send the signals. 3) The baseband signals are first mixed at $1.3 \mathrm{GHz}$ as an intermediate frequency (IF). It is then transposed to any frequencies between 2 and $12 \mathrm{GHz}$ with a second mixer. The different mixing stages are all synchronized with the $10 \mathrm{MHz}$ local oscillator (LO). The operating central frequency of the

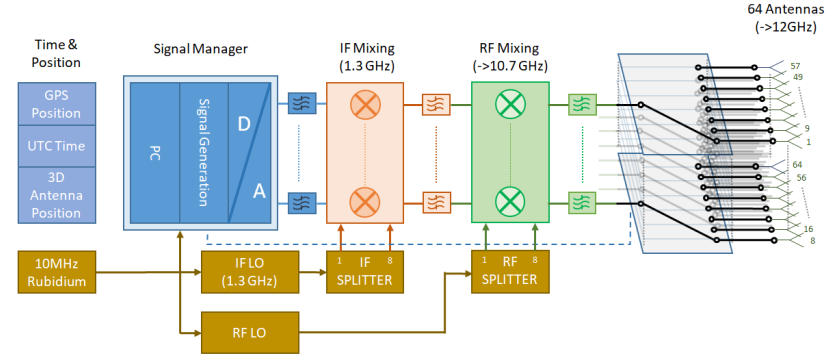

Fig. 2. MaMIMOSA Transmitter (Tx) Chain.

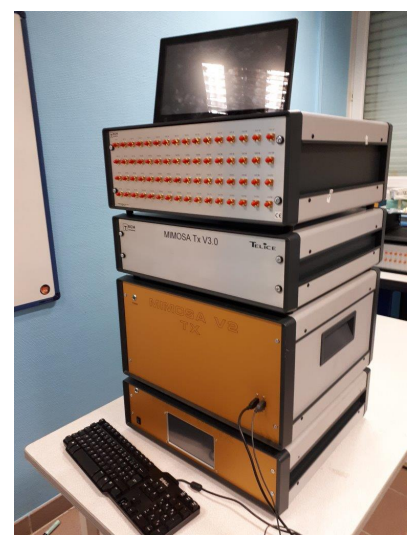

(a)

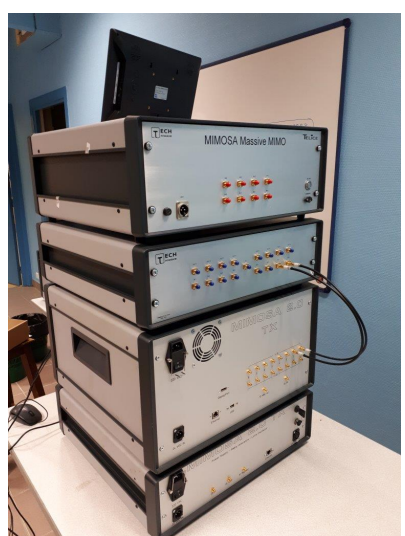

(b)
Fig. 3. Picture of the MaMIMOSA Tx Unit from the (a) front and (b) back.

sounder is selected by the user and depends on the RF filters and antennas. The maximum bandwidth is $100 \mathrm{MHz}$ with output power values which can be selected between $0.01 \mathrm{~W}$ to $1 \mathrm{~W}$ per RF chain (maximum power of $8 \mathrm{~W}$ for the 8 outputs). Figure 3 presents a front and back picture of MaMIMOSA highlighting the compactness of the sounder. The two bottom chassis form the basis of MIMOSA (operating at $1.35 \mathrm{GHz}$ ) whereas the two top chassis extend it to a massive system. The top chassis is the switching box with 8 inputs (back) and 64 outputs (front) which are controlled via the FPGA. The insertion losses are $\sim 1.7 \mathrm{~dB}$ with a switching time of 100 ns and $40 \mathrm{~dB}$ isolation between ports. The chassis beneath includes the filters and second RF mixer. A tablet can be used with a keyboard and mouse to configure the unit and visualize the selected transfer functions or power delay profiles for example.

2) Rx Unit: Similarly, the Rx chain is composed of several parts (Fig. 4). The RF module transposes the received signals to the IF module and then to baseband. The AGC adjusts the signal strength to optimize the signals at the input of the analog-to-digital converter (ADC). The different mixing stages are all synchronized with the $10 \mathrm{MHz}$ LO. The digital signals are then processed by the digital processing unit to compute in real-time the complex transfer function $\mathbf{H}(f)$ via the FPGA cards. The data is sent to an embedded PC either for display or record on a 300 GB hard drive. The GPS position and $3 \mathrm{D}$ antenna array orientation are tagged along the data. 


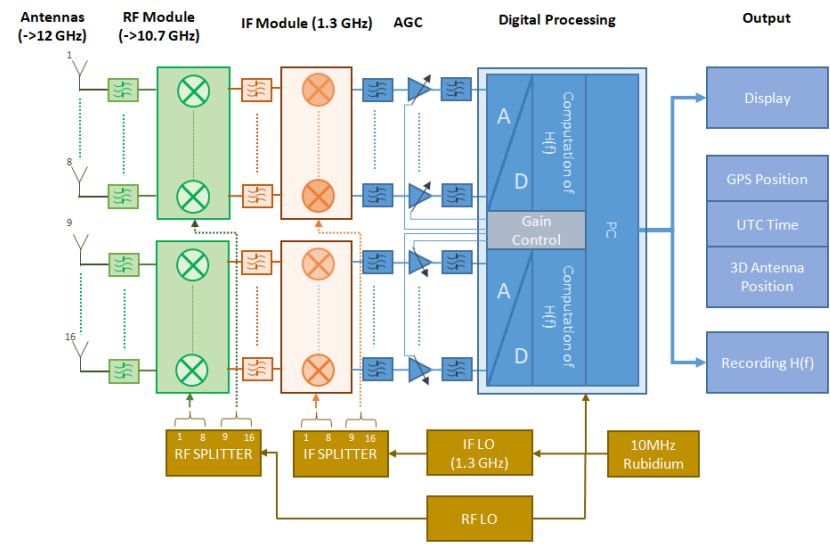

Fig. 4. MaMIMOSA Receiver (Rx) Chain.

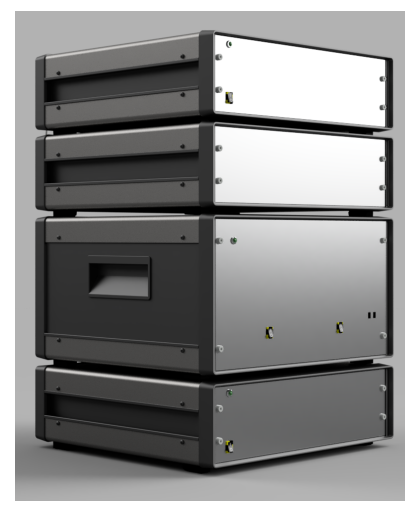

(a)

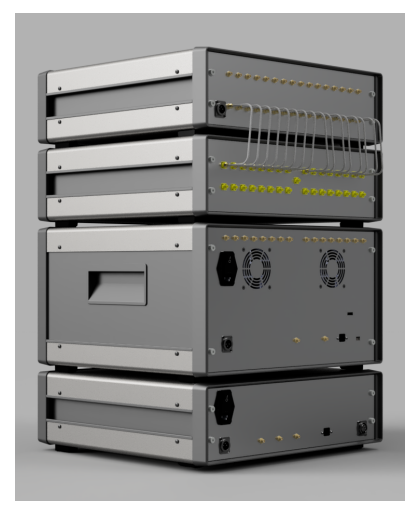

(b)
Fig. 5. 3D rendering of the MaMIMOSA Rx Unit from the (a) front and (b) back.

A rubidium reference clock synchronizes all signals like for Tx. The receiver has 16 inputs such that the processing is performed in parallel. The bandwidth is $100 \mathrm{MHz}$ and the AGC dynamic is $64 \mathrm{~dB}$ with $0.5 \mathrm{~dB}$ step. Figure 5 presents a $3 \mathrm{D}$ rendering of the front and back of the Rx unit. This will be completed by the end of the year. The two top boxes are the RF to IF transposition and filters chassis, respectively. The two bottom chassis is the Rx unit of MIMOSA.

3) Calibration procedure: The calibration procedure is automatically performed in eight consecutive steps by using a $8 \times 1$ combiner placed after the Tx output switches connected to a 1 x 16 splitter placed before the Rx inputs. Each step corresponds to a $8 \times 1$ subarray of the Massive array. The measurement cables are also included into the procedure to remove their contribution from the radio channel. The splitter transfer functions are also measured and removed. The measured 64 x 16 system transfer function is recorded in a single file. It is then used dynamically to calibrate the radio channel transfer function each time it is measured. This calibration procedure is possible because the signal sent to each emitting port is mapped to a specific set of frequencies thanks to frequency-division multiplexing.

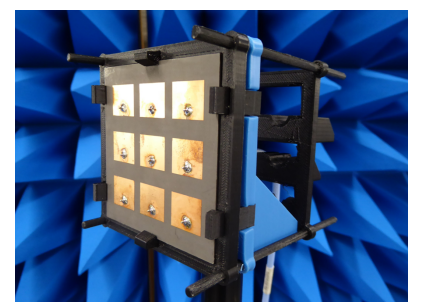

(a)

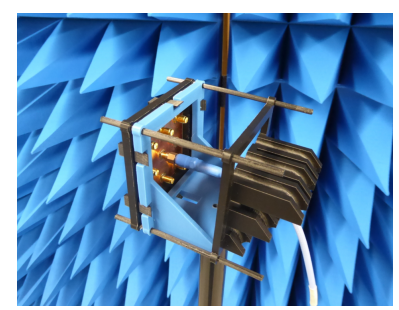

(b)
Fig. $6.3 \times 3$ array prototype for the $10 \times 10$ antenna array, (a) front and (b) back.

\section{MASSIVE MIMOSA FOR V2X COMMUNICATIONS}

\section{A. Massive antenna array and receiving antenna}

Since the target application is V2X communications, a dedicated massive antenna array operating at $5.89 \mathrm{GHz}$ with $80 \mathrm{MHz}$ bandwidth is considered. The array will be sitting on a tripod and will primarily be used as a static access point for V2I scenarios. The MaMIMOSA antenna array will be a vertical planar array of $10 \times 10$ antennas and is currently being designed and built by the University of Ghent. Since 64 elements of the array can be connected via SMA coaxial cables to the Tx unit, the remaining antennas (array perimeter) will be used as dummies and connected to $50 \Omega$ loads to attenuate the finite array side effects. The elementary element is a vertically polarized patch (duroid 5880) with $250 \mathrm{MHz}$ bandwidth at $-10 \mathrm{~dB}$ around $5.89 \mathrm{GHz}$ as shown in Fig. 7. This bandwidth much larger than the available $100 \mathrm{MHz}$ bandwidth of the sounder is needed to obtain sufficient bandwidth for the final array due to coupling effects. The plots were simulated with Sim4Life (ZMT, Zurich, Switzerland), a full-wave 3D multiphysics simulation platform. The finite-difference timedomain (FDTD) technique was used to simulate $S_{11}$ and $S_{21}$. From the optimized patch, a prototype for a $3 \times 3$ subarray has been designed and constructed. A picture of the prototype is shown in Fig. 6 when it was tested in an anechoic chamber. Finally, Fig. 8 presents a 3D rendering of the $10 \times 10$ array with the radome in transparency and support cables on the back. Since all patches of the array are mono-polarized, a $90^{\circ}$ rotation of the array will allow selecting either the vertical or horizontal polarization.

Finally, all 16 antennas that will be used at the Rx side consist in the same patch antenna than for Tx. Each antenna will be integrated into a dedicated support that can be safely attached to a high-speed moving vehicle or simply set on a table.

\section{B. Doppler characteristics of the system}

Critical features of multidimensional radio channel sounders designed to assess Doppler characteristics are the Doppler span and resolution. The former defines the maximum Doppler shift of the multipath components (MPC) whereas the latter defines the observable minimum Doppler difference between MPC. The Doppler resolution is inversely proportional to the total measurement time which is by itself constrained by the 


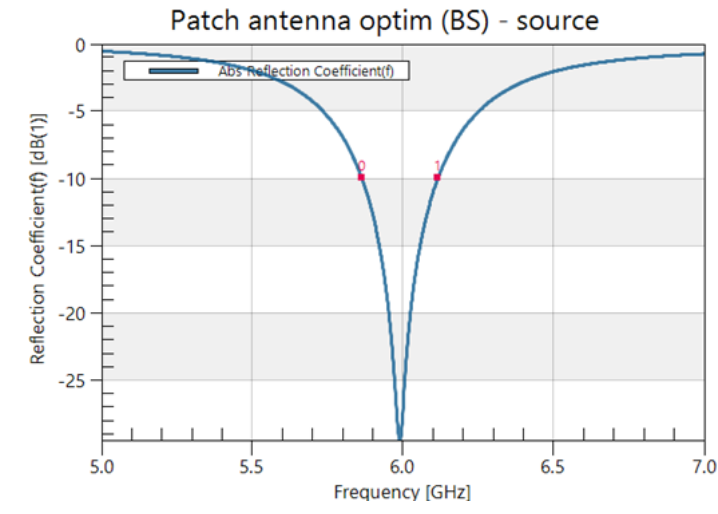

Fig. 7. Frequency characteristics of an individual patch antenna.

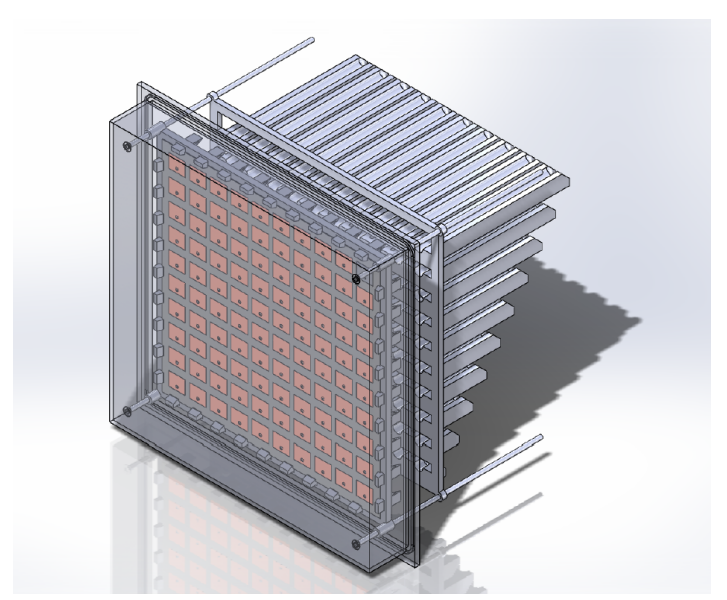

Fig. 8. 3D rendering of the final $10 \times 10$ antenna array with radome and cable support on the back.

capability of the system to treat the received signals and record the processed data on the physical hard drive. Depending on the massive MIMO radio channel configuration desired by the user and investigated vehicular scenario, the system has been designed to be as flexible as possible to select the best Doppler parameters without significant intercarrier interference. Basically, the recording time can be increased by either increasing the interframe time, intersymbol time in a frame, or decreasing the number of Rx users. The configuration parameters can be set by the user for different mobility scenarios and Doppler resolution values. Overall, the system is limited by the 100 $\mathrm{Mo} / \mathrm{s}$ data transfer from the FPGA cards to the hard drive. Evidently, the recording is also bounded by the hard drive capacity. As an example, for an urban scenario where the regulated speed is typically $50 \mathrm{~km} / \mathrm{h}( \pm 277 \mathrm{~Hz}$ maximum Doppler shift), a massive 64 x 16 MIMO channel can be measured during 120 seconds with a $1 \mathrm{~Hz}$ Doppler resolution and $300 \mathrm{~Hz}$ Doppler span. This only requires 15 Go on the hard drive and the measurement can be repeated to entirely fill the remaining space.

\section{CONCLUSION}

In this contribution, a real-time 64 x 16 massive MIMO sounder called Massive MIMOSA or MaMIMOSA based on space-frequency division multiplexing and antenna subarray switching has been presented. The sounder can operate between 2 and $12 \mathrm{GHz}$ and all signal processing is performed thanks to high-end FPGA cards at Tx and Rx giving a large possibility of antenna allocation, Doppler resolution/span, and frequency tone. Typically, the Doppler resolution can be varied from hundreds of $\mathrm{mHz}$ to a few $\mathrm{Hz}$ with equivalent speeds of a vehicle in a city or highways. For instance, for $50 \mathrm{~km} / \mathrm{h}$ maximum speeds, a 64 × 16 massive MIMO channel can be recorded as long as desired by the user with a $1 \mathrm{~Hz}$ Doppler resolution. MaMIMOSA is currently being setup to operate with a $10 \times 10$ massive antenna array at $5.89 \mathrm{GHz}$ with a 80 $\mathrm{MHz}$ bandwidth for 5G high-mobility V2X applications and will be operational in early 2020 .

\section{ACKNOWLEDGMENT}

This work was supported through the OS4 SMARTIES research program by the ELSAT2020 project co-financed by the European Union with the European Regional Development Fund, the French state, and the Hauts-de-France Region Council.

\section{REFERENCES}

[1] I. F. Akyildiz, S. Nie, S.-C. Lin, and M. Chandrasekaran, "5g roadmap: 10 key enabling technologies," Computer Networks, vol. 106, pp. $17-48,2016$. [Online]. Available: http://www.sciencedirect.com/science/article/pii/S1389128616301918

[2] C. Wang, F. Haider, X. Gao, X. You, Y. Yang, D. Yuan, H. M. Aggoune, H. Haas, S. Fletcher, and E. Hepsaydir, "Cellular architecture and key technologies for $5 \mathrm{~g}$ wireless communication networks," IEEE Communications Magazine, vol. 52, no. 2, pp. 122-130, February 2014.

[3] D. Phan-Huy, M. Sternad, and T. Svensson, "Making 5g adaptive antennas work for very fast moving vehicles," IEEE Intelligent Transportation Systems Magazine, vol. 7, no. 2, pp. 71-84, Summer 2015.

[4] A. Osseiran, F. Boccardi, V. Braun, K. Kusume, P. Marsch, M. Maternia, O. Queseth, M. Schellmann, H. Schotten, H. Taoka, H. Tullberg, M. A. Uusitalo, B. Timus, and M. Fallgren, "Scenarios for $5 \mathrm{~g}$ mobile and wireless communications: the vision of the metis project," IEEE Communications Magazine, vol. 52, no. 5, pp. 26-35, May 2014.

[5] A. Gupta and R. K. Jha, "A survey of $5 \mathrm{~g}$ network: Architecture and emerging technologies," IEEE Access, vol. 3, pp. 1206-1232, 2015.

[6] S. Parkvall, E. Dahlman, A. Furuskar, and M. Frenne, "Nr: The new $5 \mathrm{~g}$ radio access technology," IEEE Communications Standards Magazine, vol. 1, no. 4, pp. 24-30, Dec 2017.

[7] 3GPP, 3GPP Technical Report Technical Specification Group Radio Access Network; Study on NR Industrial Internet of Things (IoT);(Release 16), 2015. [Online]. Available: https://www.3gpp.org/ftp/Specs/archive/38 series/38.825.

[8] T. L. Marzetta, "Noncooperative cellular wireless with unlimited numbers of base station antennas," IEEE Transactions on Wireless Communications, vol. 9, no. 11, pp. 3590-3600, November 2010.

[9] N. B. Labs., 5G New Radio (NR) : Physical Layer Overview and Performance., 2018. [Online]. Available: http://ctw2018.ieeectw.org/files/2018/05/5G-NR-CTW-final.pdf

[10] K. Technologies., Field Testing in 5G NR., 2018. [Online]. Available: https://literature.cdn.keysight.com/litweb/pdf/5992-3299EN.pdf

[11] HUAWEI., 5 G Spectrum/ Public Policy Position., 2017. [Online]. Available: http://www-file.huawei.com/-/media/CORPORATE/PDF/publicpolicy/public policy position 5 s spectrum.pdf

[12] E. Björnson, J. Hoydis, M. Kountouris, and M. Debbah, "Massive mimo systems with non-ideal hardware: Energy efficiency, estimation, and capacity limits," IEEE Transactions on Information Theory, vol. 60, no. 11, pp. 7112-7139, Nov 2014. 
[13] J. Jose, A. Ashikhmin, T. L. Marzetta, and S. Vishwanath, "Pilot contamination and precoding in multi-cell tdd systems," IEEE Transactions on Wireless Communications, vol. 10, no. 8, pp. 2640-2651, August 2011.

[14] E. G. Larsson, O. Edfors, F. Tufvesson, and T. L. Marzetta, "Massive mimo for next generation wireless systems," IEEE Communications Magazine, vol. 52, no. 2, pp. 186-195, February 2014.

[15] M. Yusuf, E. Tanghe, F. Challita, P. Laly, D. Gaillot, M. Lienard, L. Martens, and W. Joseph, "Stationarity analysis of v2i radio channel in a suburban environment," IEEE Transactions on Vehicular Technology, pp. 1-1, 2019.

[16] J. Vieira, S. Malkowsky, K. Nieman, Z. Miers, N. Kundargi, L. Liu, I. Wong, V. Öwall, O. Edfors, and F. Tufvesson, "A flexible 100-antenna testbed for massive mimo," in 2014 IEEE Globecom Workshops (GC Wkshps), Dec 2014, pp. 287-293.

[17] P. Laly, D. P. Gaillot, M. Lienard, P. Degauque, E. Tanghe, W. Joseph, and L. Martens, "Flexible real-time mimo channel sounder for multidimensional polarimetric parameter estimation," in 2015 IEEE Conference on Antenna Measurements Applications (CAMA), Nov 2015, pp. 1-3. 